\title{
Network Effectiveness Evaluation Method of Tactical Internet Based on QoS Performance
}

\author{
Hongling $\mathrm{Li}^{1, \text { a }}$,Yingying Chen ${ }^{1, b}$,Bingyi Qiu ${ }^{2, c}$ \\ ${ }^{1}$ Department of Information Communication, Army Academy of Armored Forces, Beijing 100072, \\ China; \\ 2 63888Troops, Jiyuan 400090, China.
}

alibian0503@163.com, bwhchyingy@163.com, c11761271@qq.com

\begin{abstract}
According to the characteristics of Tactical Internet architecture, a effectiveness evaluation method of Tactical Internet based on QoS performance is proposed. The performance of the whole network is evaluated by using the underlying link transmission quality. It solves the difficult problems of evaluation caused by the different characteristics of Tactical Internet subnets and different network protocols.
\end{abstract}

Keywords: Tactical Internet, network efficiency, evaluation, index system.

\section{Introduction}

As the main communication means below brigade, Tactical Internet is more and more widely used in the future combat training. In the past, the evaluation methods were mainly focused on the single devices performance in the network, while the effectiveness evaluation methods for the whole Tactical Internet were not much studied. However, with the rapid improvement of the actual combat requirements of the troops, on the basis of satisfying the performance index of single equipment, it has become a key issue in troop training and equipment test in the future how evaluates the overall operational effectiveness of the Tactical Internet in the use of the formed system.

At present, there are many research literatures for Tactical Internet, and more in-depth research results have been obtained [1 3]. According to the characteristics of Ad hoc network, the evaluation of the interference effect is emphatically analyzed based on the analysis of the protocol and standard between American SINCGARS radio stations in literature [2]. In document [3], the protocols commonly used in MANET are analyzed such as AODV, DSR and DSDV. And the network simulation models are built with these protocols to evaluate the network effectiveness with these protocols. Although these documents give more valuable references, because of the different systems Characteristics of the Tactical Internet, it is difficult to evaluate the network efficiency of the whole network simply by only one method. In this paper, a network effectiveness evaluation method based on QoS (Quality of Service) performance is proposed in order to evaluate network effectiveness with the lowest link test value and which can be provided reference for Tactical Internet applications.

\section{The Composition and Characteristics of Tactical Internet}

The definition of Tactical Internet is given in the latest version of military language [4 5]: a mobile tactical communication system with network interconnection protocol (such as IP) which integrates all kinds of communication networks, such as tactical radio network, field integrated service digital network, system and information terminal equipment. In short, Tactical Internet is composed of Tactical Radio Internet and Field Integrated Services Digital Network, in which Tactical Radio Internet includes short wave radios, ultra-short-wave radios and high-speed data station.

From the above definition of the Tactical Internet, we can see that the Tactical Radio Internet and the Field Integrated Services Digital Network (referred to by the US military as the Field Area Communication Network) belong to different networks, and there are great differences between network architectures, protocols and organization forms etc. For example, Tactical Radio Internet is Ad hoc network structure, and its application is the incidental access of tactical subnet. The Field 
Integrated Services Digital Network is a backbone network with ATM switch. For the whole network effect evaluation method, only consider one of them is biased.

There are many network analysis and research papers on QoS performance [6 7]. Using QoS performance to evaluate the effectiveness of Tactical Internet, the different heterogeneous network evaluation is classified as the underlying data transmission performance. Furthermore, the performance of the whole network can be concluded by this way.

\section{Construction Method of Index System Based on Qos}

The index system is the key to the evaluation. The index system of network based on QoS performance mainly uses the evaluation index of link transmission quality to construct.

\subsection{Index System Based on Qos}

From the application level, whether it is the sub network of ultra-short wave radio, the sub network of short wave radio, the subnet of high speed data station in the Tactical Tadio Tnternet or The Field Integrated Services Digital Network, the evaluation of the single subnet in the Tactical Internet mainly includes the transmission speed, the transmission reliability and the transmission accuracy, and the single network efficiency can be obtained from these aspects. The evaluation index system is shown in Table 1.

Table 1. Performance index of single network

\begin{tabular}{l|c|c}
\hline \multirow{4}{*}{ Network Qos performance } & Performance index & Qos parameters \\
\cline { 2 - 3 } & \multirow{2}{*}{ Transmission speed } & IP packet transmission delay (IPTD) \\
\cline { 2 - 3 } & & IP packet delay vibration (IPDV) \\
\cline { 2 - 3 } & Transmission accuracy & IP packet error rate (IPER) \\
\cline { 2 - 3 } & Transmission reliability & IP packet loss rate (IPLR) \\
\hline
\end{tabular}

(1) IP packet transmission delay (IPTD)

In the network, the IPTD is the end-to-end transmission delay of all the successful data packets which can be calculated in the whole simulation process. For any data packet message, the time interval between the time when the source node is sent out and it when the packet is successfully received by the destination node is the specific end-to-end delay of the data transmission. It includes routing time, packet forwarding time and packet queue waiting time in seconds. The smaller the average end-to-end delay, the faster the transmission speed of the network, the better the performance of sending data packets.

(2) IP packet delay vibration (IPDV)

The IPDV is defined as the one-way delay difference between two packets in the same data stream. In the application of packet transmission, the merits and demerits of the network retransmission mechanism can be measured according to the variation of IP packet delay, which is related to the link bandwidth and the link utilization rate and is an important index to evaluate the link access performance.

(3) IP packet error rate (IPER)

The IPER is the ratio of the result of error IP packet transfer to the sum of error IP packet and successful IP packet transmission.

(4) IP packet loss rate (IPLR)

The IPLR is the ratio of the lost IP packet results to all IP packets transmission and is complementary to the packet delivery rate. The greater the value of the packet delivery rate, the less the number of data packets dropped in the transmission process, the better the performance of the routing protocol. The grouping of the parameter statistics refers to the user data packet, not including the Routing control message packet. 


\subsection{Test Method of Index System}

Define SCR as the sending point and DST as the receiver and test the value of each index according to the following method.

(1) the value of IPTD

1)SCR and DST synchronization

2)Send 100 IP packets with timestamp and group number identification on SRC side

3) If the test packet arrives at DST within the appropriate time, the time identity corresponding to the arrival of the IP packets is recorded immediately, and the one-way delay is calculated by the difference between the two-time identifiers.

(2) the value of IPDV

Two packets will be selected in the packet flow from SCR to DST. Time T1 is the first bit of SCR sending the first packet, and time T2 is the first bit of SCR sends the second packet, as shown in Fig.1. The value of the IPDV can be got by dT2-dT1.

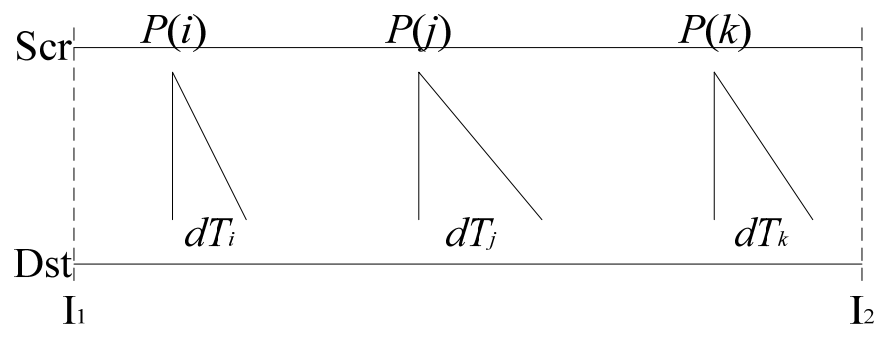

Fig 1. IP packet delay vibration

(3) the value of IPER

1) Synchronize SRC and DST.

2) Send 100 IP packets to DST with time ID and group number identification on SRC.

3) If the IP packet arrives at DST within the appropriate time, record the IP packet time ID and group number.

4) Compare corresponding number of IP packets with SRC and DST and record the number of packets that are inconsistent with the relevant content, ER.

5) IPER $=$ ER / 100.

(4) the value of IPLR

1) Synchronize SRC and DST.

2) Send 100 IP packets to DST with time ID and group number identification on SRC.

3) If the IP packet arrives at DST within the appropriate time, then record the number of IP packets arriving at DST, N.

4) The number of lost packets $P L=100-\mathrm{N}$.

5) $\mathrm{IPLR}=\mathrm{PL} / 100$.

\subsection{Network Link Weight Analysis}

On the basis of obtaining the index test data, the importance of each node and link should be determined. In this paper, the weight of each node and link can be determined by the method of node contraction.

Fig. 2 is a network topology diagram. Node Vi contraction means that all the Ki nodes connected to node $\mathrm{Vi}$ are connected to node Vi short, that is to say, a new node Vi undefined is used to replace the $\mathrm{Ki}+1$ node, and the previously associated edges are now associated with the new node. 


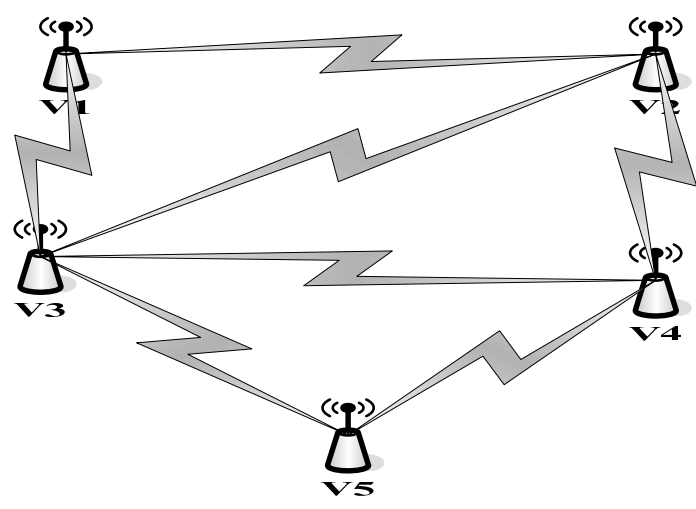

Fig 2. Network topology diagram

The schematic diagram of the shrinking nodes is shown in Fig.3.

((p))

(a)

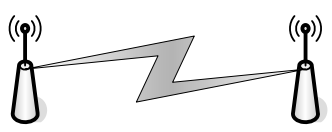

(b)

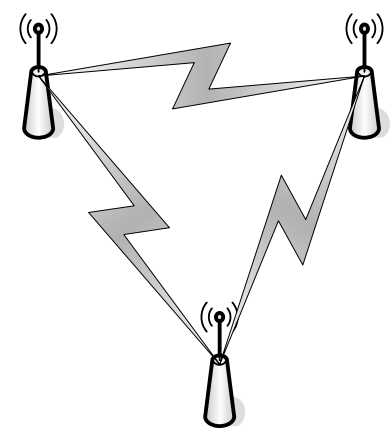

(c)

Fig 3. The diagram after the node shrinks

The graph(a) is the node V3 after contraction, and (b)is the graph of node V2 or V4 after contraction, (c) is the graph of node V1 or V5. According to the graph of node contraction, we can get the result of node importance as shown in Table 2.

Table 2. Significance assessment results of each node

\begin{tabular}{c|c|c|c|c|c}
\hline node & V1 & V2 & V3 & V4 & V5 \\
\hline Node weight & 0.3333 & 0.5 & 1 & 0.5 & 0.3333 \\
\hline
\end{tabular}

The link importance $\mathrm{KX}$ is as follows:

$$
\mathrm{Kx}=(\mathrm{Vi}+\mathrm{Vj}) / 2
$$

$\mathrm{Vi}, \mathrm{Vj}$ is the weight of two nodes that constitute the KX link.

On the basis of obtaining the above indexes value test and the weights of nodes and links, the single network can be evaluated by using numerical weighting method.

\section{Evaluation Method}

In order to obtain the statistical value of the same type of index in the whole network, the weighted fusion method is adopted after measuring the index statistics of a certain link in a certain time.

First, the link weight is normalized (suppose there are $\mathrm{n}$ test links), as shown below.

$$
\text { úx }=K_{x} / \sum_{x=1}^{n} K_{x}
$$

Then the total network statistics of a certain type of index are calculated as follows.

$$
\mathrm{IP}=\sum_{x=1}^{n} \mathrm{u} \mathrm{x} \bullet \mathrm{IPx}
$$


Using historical dates to construct the evaluation standard of network index grade. As shown in Table 3.

Table 3. Network index evaluation grade

\begin{tabular}{c|c|c|c|c}
\hline & \multicolumn{4}{|c}{ QoS level } \\
\hline The index of network performance & Level 0 & L level & Level 2 & Level 3 \\
\hline IPTD & $150 \mathrm{~ms}$ & $400 \mathrm{~ms}$ & $1 \mathrm{~s}$ & $>1 \mathrm{~s}$ \\
\hline IPDV & $50 \mathrm{~ms}$ & $50 \mathrm{~ms}$ & $1 \mathrm{~s}$ & $>1 \mathrm{~s}$ \\
\hline IPLR & & $1 \times 10^{-3}$ & $>1 \times 10^{-3}$ \\
\hline IPER & \multicolumn{3}{|c|}{$1 \times 10^{-4}$} & $1 \times>10^{-4}$ \\
\hline
\end{tabular}

According to the evaluation level given in Table 3, the performance of the whole network can be evaluated.

\section{Summary}

A method for evaluating the effectiveness of Tactical Internet based on QoS performance is presented, which provides a more effective evaluation method for the equipment construction and army application of Tactical Internet. Of course, there is still a lot of works to evaluate the effectiveness of Tactical Internet, including the improvement of network performance index system, such as whether the whole network evaluation has included all the indicators reflecting the performance of the whole network; The whole network efficiency evaluation algorithm optimization and so on, all these work needs the following further research.

\section{References}

[1]. Wang yuan. Research on the method for evaluating the resilience of Tactical Internet based on network topology [J]. Information communication, 2015, 6th issue: 41-42.

[2]. Chen Zhilin et al. Evaluation of the effect of Tactical Internet interference [J]. Fire and command control, 2016,41 (4): 126 130.

[3]. Wang Shuai et al. Application simulation and evaluation of Tactical mobile self-organizing network [J]. Journal of the institute of ordnance engineering, 2017,29 (3): 54-58.

[4]. Military language of the people's liberation army of China [M]. Beijing: military science press, 2011.

[5]. Huo jinghe, li hongling. Tactical communication system [M]. Beijing: national defense industry press, 2016.

[6]. Ye xi. Research on the key technologies for security and reliability of multimedia sensor networks supporting QoS [D]. Doctoral thesis of Nanjing university of posts and telecommunications, 2015.

[7]. Zhang Yuanyuan. Research on key technologies of heterogeneous networks based on QoS guarantee [D]. Doctoral thesis of Beijing university of technology, 2016. 